BROWN-HET-940(Rev.)

SNUTP - 94 - 59

August 1995

\title{
THE W-BOSON MASS AND PRECISION TESTS OF THE STANDARD MODEL
}

\author{
Kyungsik Kang \\ Department of Physics, Brown University, Providence, RI 02912, USA,] \\ and \\ Sin Kyu Kang \\ Department of Physics, Seoul National University, Seoul, Korea Đ
}

\begin{abstract}
We have examined the electroweak radiative corrections in the LEP precision data in view of the new measurements of $M_{W}$ and $m_{t}$ as well as the recent progress in the higher order radiative corrections. From the minimal $\chi^{2}$-fit to the experimental Z-decay parameters (with the aid of a modified ZFITTER program), we predict that $M_{W}=80.29(4)(2) \mathrm{GeV}$ where the first error is due to the uncertainty in the fitted $m_{t}$ for a fixed $m_{H}$ and the second error comes from the $m_{H}$ in the range of $60-1000 \mathrm{GeV}$, which is to be compared with the current world average $M_{W}=80.23(18) \mathrm{GeV}$. The current world average value of $M_{W}$ and the 1994 LEP data definitely favor nonvanishing electroweak radiative corrections and are consistent with a heavy $m_{t}$ as measured by the recent CDF report but with a heavy Higgs scalar of about $400 \mathrm{GeV}$ within the context of the minimal standard model. The sensitivity of and the errors in the best fit solutions due to the uncertainties in the gluonic coupling $\alpha_{s}\left(M_{Z}\right)$ and $\alpha\left(M_{Z}\right)$ are also studied carefully. In addition we discuss how the future precision measurements of $M_{W}$ can provide a decisive test for the standard model with radiative corrections and give a profound implication for the measurement of t-quark and Higgs masses.
\end{abstract}

\footnotetext{
${ }^{1}$ Supported in part by the USDOE contract DE-FG02-91ER40688-Task A.

2 Supported in part by the Basic Science Research Institute program, Ministry of Education, 1994, Project No.BSRI-94-2418, and the Korea Science and Engineering Foundation through SNU CTP.
} 
Much interests have been paid in recent years to the electroweak radiative corrections (EWRC) and precision tests of the standard model thanks to the accurate data obtained at LEP [1-7]. There have been numerous articles published on the subject as has been documented in [7-9]. The LEP data are generally regarded as the support for the standard model and as the evidence of the nonvanishing EWRC [9].

However there have been several important developments since last year which warrant a new motivation to repeat the precision tests of the standard model. Some of the experimental advances are (1) the new CDF value for $M_{W}$ [10], (2) the improved LEP data [11], and (3) the CDF and D0 reports [5 on $m_{t}[12]$, while there have been also some progress on the higher order corrections, in particular the dominant two-loop terms of order $\alpha^{2} m_{t}^{4}$ [14], the QCD-electroweak mixed diagrams [14,15] and higher order corrections to the QCD factor in the $Z$ - decay width [16]. We would like to present the results of the new fit to the updated 1994 data with the aid of the appropriately modified ZFITTER program to incorporate these new theoretical developments. We examine the errors in the best fit solutions due to the uncertainties in the strong coupling constant $\alpha_{s}\left(M_{Z}\right)$ and also in $\alpha\left(M_{Z}\right)$. In the analysis we determine $M_{W}$ self-consistently from the W-mass relation that includes EWRC for the value of $m_{t}$ covering experimental range and fit the LEP data, and show how stable the predicted $M_{W}$ is regardless the exact value of $m_{H}$ in the interesting range of $60-1000 \mathrm{GeV}$. Though the sensitivity of the EWRC to the exact value of $M_{W}$ in the standard model has been studied based on the $W$-mass formula [17], the effect of the self-consistency satisfied by $M_{W}$ through the mass relation with EWRC as well as that of the uncertainties in $\alpha_{s}\left(M_{Z}\right)$ and $\alpha\left(M_{Z}\right)$ to the precision tests of the electroweak data has not been fully examined and understood. For this reason, we would like to critically examine in this paper the sensitivity of the precision tests and the $m_{t}-m_{H}$ correlation to the requirement of consistency in the needed EWRC for a set of mass values as well as the errors in $\alpha_{s}\left(M_{Z}\right)$ and $\alpha\left(M_{Z}\right)$. In particular, the results of the minimal $\chi^{2}$-fit show that the $\mathrm{CDF}$ value $m_{t}=174 \mathrm{GeV}$ can be consistent with a best fit solution but with a Higgs mass about $m_{H}=400 \mathrm{GeV}$ if $\alpha_{s}\left(M_{Z}\right)=0.123$ and $\alpha^{-1}\left(M_{Z}\right)=128.87$ and predict $M_{W}=80.29(4)(2) \mathrm{GeV}$ where the first error is due to the uncertainty in the fitted $m_{t}$ for a fixed $m_{H}$ and the second error comes from the $m_{H}$ in the range $60-1000 \mathrm{GeV}$. However $m_{t}$ and $m_{H}$ can easily be shifted, due to the uncertainty in the gluonic coupling $\Delta \alpha_{s}= \pm 0.006$, by as much as $5 \mathrm{GeV}$ and $125 \mathrm{GeV}$ respectively, while the corresponding shift in $M_{W}$ turns out to be about $30 \mathrm{MeV}$. The possible shifts in $m_{t}, m_{H}$ and $M_{W}$ due to the error in the gauge coupling $\Delta \alpha^{-1}\left(M_{Z}\right)= \pm 0.12$ can be as much as $6 \mathrm{GeV}, 160 \mathrm{GeV}$ and $20 \mathrm{MeV}$ respectively.

\footnotetext{
${ }^{3}$ The most recent values [13] are $m_{t}=176 \pm 8 \pm 10 \mathrm{GeV}(\mathrm{CDF})$ and $m_{t}=199_{-21}^{+19} \pm 22 \mathrm{GeV}$ (D0).
} 
In addition, we reexamined the claim made by Novikov, Okun, and Vysotsky [18] that the 1993 data from LEP on the electroweak parameters as defined in the standard model could be explained by the QED Born approximation (QBA) in which $\alpha\left(M_{Z}\right)$ is used instead of $\alpha(0)$ in the tree approximation along with the corresponding redefinition of the weak mixing angle $\sin ^{2} \theta$ instead of $\sin ^{2} \theta_{W}$. In particular the so-called QBA predictions were claimed to be within $1 \sigma$ accuracy of all electroweak precision measurements made at LEP in 1993. In order to examine the intriguing claim made in Ref.[18], we considered the case of the QBA by consistently neglecting the terms of non-photonic origin in the full EWRC. The full EWRC is calculated with the aid of a modified ZFITTER program [19] that uses an improved QCD correction factor [16], includes the dominant two-loop and QCD-electroweak terms [14] and makes the minimal $\chi^{2}$ fit to the data. We have found [21] that the situation is very sensitive to the value of $M_{W}$ and that the QBA fit to the 1993 data gives statistically comparable or better $\chi^{2}$ and therefore the existence of the genuine quantum effect due to full EWRC might not have been evident in the 1993 data. We note that the old $M_{W}$ has somewhat lower central value and larger error. The new results of the minimal $\chi^{2}$ fit to the updated 1994 data and the predicted $M_{W}$ compared to the new world average $M_{W}$ show a clear effect due to the EWRC.

It is well known that the charge renormalization in the conventional QED fixes the counter term by the renormalized vacuum polarization $\hat{\Pi}^{\gamma}(0)$ and one can evaluate $\hat{\Pi}^{\gamma}\left(q^{2}\right)=\hat{\Sigma}^{\gamma \gamma}\left(q^{2}\right) / q^{2}$ from the photon self energy $\hat{\Sigma}^{\gamma \gamma}\left(q^{2}\right)$, for example, by the dimensional regularization method. This gives at $q^{2}=M_{Z}^{2}$ the total fermionic contribution of $m_{f} \leq$ $M_{Z}$ to the real part $\operatorname{Re} \hat{\Pi}^{\gamma}\left(M_{Z}^{2}\right)=-0.0596(9)$, which includes both the lepton and quark parts [22]. Here, the quark contribution to $\operatorname{Re} \hat{\Pi}^{\gamma}\left(q^{2}\right)$ is the hadronic one which can be directly evaluated by dispersion integral over the measured cross section of $e^{+} e^{-} \rightarrow$ hadrons. Then, we get $\alpha\left(M_{Z}\right)=1 / 128.87(12)$ in the on-shell scheme if the hyperfine structure constant $\alpha=e^{2} / 4 \pi=1 / 137.0359895(61)$ is used, which we will use in this paper. The error in $\alpha\left(M_{Z}\right)$ is due to the uncertainty in hadronic contribution. This is obviously a source of the uncertainty in the best fit solutions.

The electroweak parameters are evaluated numerically with the hyperfine structure constant $\alpha$, the four-fermion coupling constant of $\mu$-decay, $G_{\mu}=1.16639(2) \times 10^{-5} \mathrm{GeV}^{-2}$, and $Z$-mass $M_{Z}=91.1888(44)$ in the 1994 data fit. Numerical estimate of the full EWRC requires the mass values of the leptons, quarks, and Higgs scalar besides these quantities. While $Z$-mass is known to an incredible accuracy from the LEP experiments largely due to the resonant depolarization method, the situation with respect to the $W$-mass is

\footnotetext{
${ }^{4}$ We note that the higher QCD effects $\left(\alpha \alpha_{s}^{2} m_{t}^{2}\right.$ order) are yet to be unanimously agreed by the experts as evidenced by the discussions in $[15,20]$.
} 


\begin{tabular}{|c|c|c|c|}
\hline \hline & $M_{W}(\mathrm{GeV})$ & $\Delta r$ & $\sin ^{2} \theta_{W}$ \\
\hline 1 & 79.91 & 0.0623 & 0.2321 \\
\hline 2 & 80.22 & 0.0448 & 0.2261 \\
\hline 3 & 80.23 & 0.0443 & 0.2263 \\
\hline 4 & 80.38 & 0.0355 & 0.2231 \\
\hline \hline
\end{tabular}

Table 1: Dependence of the radiative correction $\Delta r$ on the values of $M_{W}$.

desired to be improved, i.e., $M_{W}=80.22(26) \mathrm{GeV}[23]$ and $80.23(18) \mathrm{GeV}[10]$ from the world average values vs. the old and new CDF measurements $M_{W}=79.91(39) \mathrm{GeV}$ [24] and $M_{W}=80.38(23) \mathrm{GeV}[10]$. The minimal $\chi^{2}$-fit to the LEP data will at best give the $m_{t}-m_{H}$ correlations as shown in Fig. 1(a) and Fig. 1(b). We will show how the best fit solutions are chosen out of the solution set for $\left(m_{t}, m_{H}\right)$ and determine $M_{W}$ self-consistently from the $W$-mass relation that includes EWRC and how they depend on the uncertainties in $\alpha_{s}\left(M_{Z}\right)$ and $\alpha\left(M_{Z}\right)$ so that how precisely the standard model can be tested at the moment, let alone the predictions of the $m_{t}-m_{H}$ correlation from the experimental $M_{W}$.

One has, in the standard model, the on-shell relation $\sin ^{2} \theta_{W}=1-\frac{M_{W}^{2}}{M_{Z}^{2}}$, while the four-fermion coupling constant $G_{\mu}$ can be written as

$$
G_{\mu}=\frac{\pi \alpha}{\sqrt{2} M_{W}^{2}}\left(1-\frac{M_{W}^{2}}{M_{Z}^{2}}\right)^{-1}(1-\Delta r)^{-1}
$$

so that $\Delta r$, representing the radiative corrections, is given by

$$
\Delta r=1-\left(\frac{37.2802}{M_{W}}\right)^{2} \frac{1}{1-M_{W}^{2} / M_{Z}^{2}} .
$$

We note from Table 1 that the radiative correction $\Delta r$ is very sensitive to the value of $M_{W}$. Mere change in $M_{W}$ by $0.59 \%$ results as much as a $43 \%$ change in $\Delta r$. Theoretically, the radiative correction parameter $\Delta r$ within the standard model can be written as [25]

$$
1-\Delta r=(1-\Delta \alpha) \cdot\left(1+\cot ^{2} \theta_{W} \Delta \bar{\rho}\right)-\Delta r_{r e m},
$$

where $\Delta \bar{\rho}$ contains one loop and the leading 2-loop irreducible weak and QCD corrections. Main contribution to $\Delta \bar{\rho}=1-\rho^{-1}$ is from the heavy t-quark through the mass renormalizations of weak gauge bosons $W$ and $Z$, while there is a part in $(\Delta r)_{r e m}$ containing also the t-quark and Higgs scalar contributions. Note that the so-called QBA to $\Delta r$ is defined by keeping only the photon vacuum polarization contribution, $\Delta \alpha=-\operatorname{Re} \hat{\Pi}^{\gamma}\left(M_{Z}^{2}\right)=0.0596$. We see from Table 1 that $\Delta \alpha$ is numerically the dominant component of the radiative 
corrections. In particular for the old CDF $M_{W}, \Delta \alpha$ is already within $4.4 \%$ of the needed $\Delta r$ and is close enough to be within the experimental uncertainty. However, with the current world average value $M_{W}=80.23 \mathrm{GeV}, \Delta \alpha$ differs by $32 \%$ from the needed $\Delta r$ that has to be accounted for by the weak interaction corrections [26].

Note that precise determination of the on-shell value of $\sin ^{2} \theta_{W}$ can also constrain the needed radiative correction and the value of $M_{W}$.

We have searched for the minimal $\chi^{2}$-fits to both 1993 and earlier 1994-data of the Zdecay parameters measured at LEP by using the modified ZFITTER program [21]. Within the framework of the standard model in which $G_{\mu}, \alpha$ and $M_{Z}$ are taken as input, one can predict $M_{W}$ from (2) in a self-consistent manner. Starting with the given masses of the quarks, leptons, gauge bosons and Higgs scalar, as well as given gluonic coupling $\alpha_{s}\left(M_{Z}\right)$ and gauge coupling $\alpha\left(M_{Z}\right), \Delta r$ is calculated from (3) by including up to the dominant two-loop and QCD-electroweak mixed terms and then is used to determine $M_{W}$ from the right hand side of (2). With this new $M_{W}, \Delta r$ is calculated again to determine another new $M_{W}$. This iteration process is repeated until $\Delta r$ converges to within $O\left(10^{-6}\right)$. The final output $M_{W}$ from this iteration procedure is the self-consistent solution of (2) for $M_{W}$ with the starting set of $m_{t}, m_{H}$ etc. Upon varying $m_{t}$, this procedure will give the $m_{t}-M_{W}$ correlation for all other parameters including $m_{H}$ fixed. The family of the curves in Fig. 2 represents such correlations for different fixed values of $m_{H}$. The $m_{t}-m_{H}$ correlation shown in Fig. 1(a) and Fig. 1(b) is not restrictive enough to discriminate an interesting region of $m_{t}$ and $m_{H}$ when compared to the experimental range of $m_{t}$. Also the $M_{W}-m_{t}$ correlation shown in Fig. 2 does not discriminate $m_{H}$ as long as it is heavier than 100 $\mathrm{GeV}$ given the current experimental situation of $\left(m_{t}, M_{W}\right)$. Fig. 3 shows the result of the self-consistent procedure for $M_{W}-m_{H}$ correlation when $m_{t}$ is restricted to the CDF value $174 \pm 16 \mathrm{GeV}$ on which the predicted $M_{W}$ range is also indicated. The error band \pm 0.04 $\mathrm{GeV}$ in the predicted $M_{W}$ is due to the uncertainty in the fitted $m_{t}$ for a fixed $m_{H}$ as one can deduce from Table 2 . We then calculate the eleven $Z$-decay parameters, as chosen in Table 2, for the parameter sets $\left(m_{t}, m_{H}\right)$ that determine $M_{W}$ from (2) and search for the minimal $\chi^{2}$-fit solution to the experimental $Z$-decay parameters. This procedure selects the Best.fit curve in Fig. 1 and $\diamond$ points on each curve in Fig. 2. Including the error due to varying $m_{H}$ in the range, we predict $M_{W}=80.29(4)(2) \mathrm{GeV}$, which is slightly larger than the central value of the current world average $80.23 \mathrm{GeV}$. Note that the predicted $M_{W}$ constrains $m_{H}$ to lie $230 \mathrm{GeV}$ and $830 \mathrm{GeV}$ for $m_{t}=174 \pm 16 \mathrm{GeV}$ as one can see from Fig.3.

The results of the best $\chi^{2}$-fits to the updated 1994 data are given for the four sets of $\left(m_{t}, m_{H}\right)$ in Table 2. The $Z$-decay parameters are calculated with the gluonic 
coupling constant in the range $\alpha_{s}\left(M_{Z}\right)=0.123(6)$ in the QCD correction factor $R_{\mathrm{QCD}}=$ $1+1.05 \frac{\alpha_{s}}{\pi}+0.9( \pm 0.1)\left(\frac{\alpha_{s}}{\pi}\right)^{2}-13.0\left(\frac{\alpha_{s}}{\pi}\right)^{3}$ for light quarks(u,d,c,s) [27] and in $R_{\mathrm{QCD}}=$ $1+c_{1}\left(m_{b}\right) \frac{\alpha_{s}}{\pi}+c_{2}\left(m_{t}, m_{b}\right)\left(\frac{\alpha_{s}}{\pi}\right)^{2}+c_{3}\left(m_{t}, m_{b}\right)\left(\frac{\alpha_{s}}{\pi}\right)^{3}$, the $m_{b}$ and $m_{t}$ mass dependent one for b quarks [16]. The partial width for $Z \rightarrow f \bar{f}$ is given by

$$
\Gamma_{f}=\frac{G_{\mu}}{\sqrt{2}} \frac{M_{Z}^{3}}{24 \pi} \beta R_{\mathrm{QED}^{c}} R_{\mathrm{QCD}}\left(M_{Z}^{2}\right)\left\{\left[\left(\bar{v}_{f}^{Z}\right)^{2}+\left(\bar{a}_{f}^{Z}\right)^{2}\right] \times\left(1+2 \frac{m_{f}^{2}}{M_{Z}^{2}}\right)-6\left(\bar{a}_{f}^{Z}\right)^{2} \frac{m_{f}^{2}}{M_{Z}^{2}}\right\}
$$

where $\beta=\sqrt{1-4 m_{f}^{2} / M_{Z}^{2}}, R_{\mathrm{QED}}=1+\frac{3}{4} \frac{\alpha}{\pi} Q_{f}^{2}$ and the color factor $c_{f}=3$ for quarks and 1 for leptons. Here the renormalized vector and axial-vector couplings are defined by $\bar{a}_{f}^{Z}=\sqrt{\rho_{f}^{Z}} 2 a_{f}^{Z}=\sqrt{\rho_{f}^{Z}} 2 I_{3}^{f}$ and $\bar{v}_{f}^{Z}=\bar{a}_{f}^{Z}\left[1-4\left|Q_{f}\right| \sin ^{2} \theta_{W} \kappa_{f}^{Z}\right]$ in terms of the familiar notations $[19,28]$. Note that $\Delta \alpha$ is included in the couplings through $\sin ^{2} \theta_{W}$ via (1) and (3) and all other non-photonic loop corrections are grouped in $\rho_{f}^{Z}$ and $\kappa_{f}^{Z}$ as in [19,29] including the dominant two-loop and QCD-electroweak terms. Note that the QED loop corrections can unambiguously be separated from the electroweak loops in the case of neutral current interactions [28]. Thus the case of the QBA can be achieved simply by setting $\rho_{f}^{Z}$ and $\kappa_{f}^{Z}$ to 1 in the vector and axial-vector couplings.

Numerical results for the best $\chi^{2}$-fits to the 1993 LEP experimental parameters of $Z$ decay for $M_{W}=79.91(39) \mathrm{GeV}$ [24] and $M_{W}=80.22(26) \mathrm{GeV}$ [23] showed [21] generally small contributions of the weak corrections and in particular that the QBA was close to the experimental values within the uncertainty of the measurements, i.e., within $2 \sigma$. The near absence of the weak interaction contributions to the radiative corrections for the 1993 data is more impressive for $M_{W}=79.91(39) \mathrm{GeV}$ than for $M_{W}=80.22(26) \mathrm{GeV}$. This is mainly because QBA gives $M_{W}=79.95 \mathrm{GeV}$ compared to $M_{W}=80.10(1) \mathrm{GeV}$ from the full EWRC for $m_{H}$ in the range of $60-1000 \mathrm{GeV}$ and a larger error in the former as observed in $[18,21]$. At closer examination, however, the QBA in this case over-estimates the radiative corrections and the full EWRC fair better; for $M_{W}=80.22(26) \mathrm{GeV}$ and $m_{H}=60-1000 \mathrm{GeV}, \Delta r=0.0596$ in QBA and $0.0498-0.0505$ in the full EWRC to be compared to the required value 0.0448. Also the global fit to the 1993 data with two variables $m_{t}$ and $m_{H}$ in the range $60-1000 \mathrm{GeV}$ show [21] that the best fits can be achieved by $m_{t}=139(17) \mathrm{GeV}$ wth a stable output $M_{W}=80.12(1) \mathrm{GeV}$ when the full EWRC are taken into account.

The situation with the minimal $\chi^{2}$-fits to the updated 1994 LEP data and with theoretically determined $M_{W}$ is significantly different from the case of the 1993 data as one can see from Table 2. Not only there is clear evidence of the full EWRC in each of the eleven $Z$-parameters but also the best fit solutions to the 1994 data show a stable output $M_{W}=80.29(2) \mathrm{GeV}$ for $m_{H}$ in the range of $60-1000 \mathrm{GeV}$. In particular the 


\begin{tabular}{|c|c|c|c|c|c|c|}
\hline & Experiment & $\overline{\text { Born }}$ & Full EW & $\overline{\text { Full EW }}$ & $\overline{\text { Full EW }}$ & $\overline{\text { Full EW }}$ \\
\hline$m_{t}(\mathrm{GeV})$ & $174 \pm 10_{-12}^{+13}$ & & $187_{(4)(5)(5)}^{(5)(6)}$ & $177_{(4)(6)(6)}^{(5)}$ & $163_{(4)(6)(7)}^{(5)}$ & $149_{(5)(7)}^{(4)(6)}$ \\
\hline$m_{H}(\mathrm{GeV})$ & $60 \leq m_{H} \leq 1000$ & & 1000 & 500 & 200 & 60 \\
\hline$M_{W}(\mathrm{GeV})$ & $80.23 \pm 0.18$ & $79.96_{(1)}^{(2)}$ & $80.31(3)(2)$ & $80.30_{(3)}^{(2)}(2)$ & $80.28(3)_{(2)}^{(3)}$ & $80.27(3)_{(3)}^{(2)}$ \\
\hline$\Gamma_{Z}(\mathrm{MeV})$ & $2497.4 \pm 3.8$ & $2489.0_{(0.8)}^{(0.9)}$ & $2496.5_{(1.8)(0.6)}^{(2.0)(0.8)}$ & $2496.7_{(2.1)(0.8)}^{(1.9)(0.8)}$ & $2496.5_{(1.9)(0.7)}^{(2.2)(1.0)}$ & $2495.7_{(2.2)(0.8)}^{(2.4)(0.6)}$ \\
\hline$\sigma_{h}^{P}(n b)$ & $41.49 \pm 0.12$ & $41.41_{(0)}^{(0)}$ & $41.40_{(3)(0)}^{(3)(1)}$ & $41.39_{(3)(0)}^{(3)(1)}$ & $41.39_{(3)(1)}^{(3)(0)}$ & $41.38_{(3)(0)}^{(3)(0)}$ \\
\hline$R\left(\Gamma_{h a d} / \Gamma_{l \bar{l}}\right)$ & $20.795 \pm 0.040$ & $20.850_{(6)}^{(5)}$ & $20.789_{(39)(6)}^{(39)(7)}$ & $20.798_{(38)(6)}^{(40)(7)}$ & $20.809_{(39)(6)}^{(38)(7)}$ & $20.822_{(39)(7)}^{(39)(6)}$ \\
\hline$A_{F B}^{0, l}$ & $0.0170 \pm 0.0016$ & $0.0168_{(6)}^{(5)}$ & $0.0148_{(2)(1)}^{(4)(3)}$ & $0.0149_{(3)(2)}^{(2)(2)}$ & $0.0149_{(2)(1)}^{(3)(2)}$ & $0.0152_{(2)(2)}^{(2)(2)}$ \\
\hline$A_{\tau}$ & $0.143 \pm 0.010$ & $0.150_{(3)}^{(2)}$ & $0.141_{(1)(1)}^{(1)(1)}$ & $0.141_{(1)(1)}^{(1)(1)}$ & $0.141_{(1)(1)}^{(1)(1)}$ & $0.142_{(1)(1)}^{(1)(1)}$ \\
\hline$A_{e}$ & $0.135 \pm 0.011$ & $0.150_{(3)}^{(2)}$ & $0.141_{(1)(1)}^{(1)(1)}$ & $0.141_{(1)(1)}^{(1)(1)}$ & $0.141_{(1)(1)}^{(1)(1)}$ & $0.142_{(1)(1)}^{(1)(1)}$ \\
\hline$R\left(\Gamma_{b \bar{b}} / \Gamma_{h a d}\right)$ & $0.2202 \pm 0.0020$ & $0.2180_{(0)}^{(0)}$ & $0.2154_{(0)(1)}^{(1)(2)}$ & $0.2158_{(1)(2)}^{(1)(1)}$ & $0.2161_{(0)(1)}^{(1)(2)}$ & $0.2165_{(0)(2)}^{(1)(2)}$ \\
\hline$R\left(\Gamma_{c \bar{c}} / \Gamma_{h a d}\right)$ & $0.1583 \pm 0.0098$ & $0.1707_{(0)}^{(0)}$ & $0.1711_{(0)(0)}^{(0)(0)}$ & $0.1710_{(0)(0)}^{(1)(1)}$ & $0.1709_{(0)(0)}^{(1)(1)}$ & $0.1709_{(0)(1)}^{(0)(0)}$ \\
\hline$A_{F B}^{0 . b}$ & $0.0967 \pm 0.0038$ & $0.1050_{(18)}^{(17)}$ & $0.0985_{(8)(5)}^{(11)(8)}$ & $0.0987_{(8)(6)}^{(10)(6)}$ & $0.0989_{(8)(4)}^{(10)(7)}$ & $0.0998_{(7)(7)}^{(8)(6)}$ \\
\hline$A_{F B}^{0 . c}$ & $0.0760 \pm 0.0091$ & $0.0750_{(14)}^{(14)}$ & $0.0701_{(6)(4)}^{(9)(6)}$ & $0.0703_{(8)(5)}^{(6)(4)}$ & $0.0704_{(6)(3)}^{(7)(5)}$ & $0.0711_{(5)(5)}^{(6)(4)}$ \\
\hline $\sin ^{2} \theta_{\text {eff }}^{\text {lepton }}$ & $0.2320 \pm 0.0016$ & $0.2321_{(3)}^{(3)}$ & $0.2327_{(2)(2)}^{(1)(1)}$ & $0.2326_{(2)(2)}^{(1)(1)}$ & $0.2324_{(2)(1)}^{(1)(1)}$ & $0.2322_{(2)(2)}^{(1)(1)}$ \\
\hline$\chi^{2}$ & & 19.5 & 11.2 & 10.3 & 9.57 & 9.31 \\
\hline$\Delta r$ & $0.0443 \pm 0.0102$ & $0.0596_{(9)}^{(9)}$ & $0.0397_{(19)(13)(10)}^{(16)}$ & $0.0403_{(16)(13)}^{(19)(12)}$ & $0.0414_{(19)(16)}^{(14)(11)}$ & $0.0422_{(14)(12)}^{(14)(14)}$ \\
\hline
\end{tabular}

Table 2: Numerical results including full EWRC for eleven experimental parameters of the Z-decay and $M_{W}$. Each pair of $m_{t}$ and $m_{H}$ represents the case of the best $\chi^{2}$ - fit to the $1994 \mathrm{LEP}$ data for $\alpha_{s}\left(M_{Z}\right)=0.123(6)$ and $\alpha^{-1}\left(M_{Z}\right)=128.87(12)$. The numbers in () represent the errors due to $\Delta \alpha_{s}\left(M_{Z}\right)= \pm 0.006$ and $\Delta \alpha^{-1}\left(M_{Z}\right)= \pm 0.12$ respectively. $\sin ^{2} \theta_{\text {eff }}^{\text {lepton }}$ is from the measurement of $\left\langle Q_{F B}\right\rangle$. For the case of Born approxiamtion, the errors are due to $\Delta \alpha^{-1}\left(M_{Z}\right)$ only. 
QBA gives distinctively inferior $\chi^{2}(=19.5 / 11)$ for the 1994 data. Also the CDF $m_{t}=174$ $\mathrm{GeV}$ is a possible output solution with a $m_{H}$ about $400 \mathrm{GeV}$ among the many possible combinations of $\left(m_{t}, m_{H}\right)$ given by the 'Best.fit' curve in Fig. 1(a) and Fig. 1(b). As shown in Table 2, the Best.fit solutions can have errors due to the uncertainty in $\alpha_{s}\left(M_{Z}\right)$ : $m_{t}$ and $M_{W}$ may be shifted by as much as $\pm 5 \mathrm{GeV}$ and $\pm 30 \mathrm{MeV}$ respectively because of $\Delta \alpha_{s}= \pm 0.006$. The error range of the Best.fit solutions is indicated by the curves $\mathrm{A}$ and B in Fig. 1(a). There are additional comparable errors due to the uncertainty in $\alpha\left(M_{Z}\right)$ as shown in Table 2 and Fig. 1(b) : $\Delta \alpha^{-1}\left(M_{Z}\right)= \pm 0.12$ can cause another $\pm 6 \mathrm{GeV}$ and $\pm 20 \mathrm{MeV}$ respectively in $m_{t}$ and $M_{W}$. In general the $\chi^{2}$-value tends to prefer the lower $m_{t}$ and accordingly smaller $m_{H}$, though any pair of $\left(m_{t}, m_{H}\right)$ on the Best.fit curve in Fig. 1(a) and Fig. 1(b) is statistically comparable to each other. In particular the best global fits to the updated 1994 data give $m_{t}=155-187 \mathrm{GeV}$ for $m_{H}=100-1000 \mathrm{GeV}$. Most of the $Z$-parameters are stable irrespectively to the uncertainties due to $\Delta \alpha_{s}$ and $\Delta \alpha$ and in excellent agreement with the data except $R\left(\Gamma_{b \bar{b}} / \Gamma_{h a d}\right)$. Even with the mass dependent QCD factor, there is still about $2.4 \sigma$ deviation in $R\left(\Gamma_{b \bar{b}} / \Gamma_{h a d}\right)$ from the experiments irrespectively to the uncertainties in $\alpha_{s}\left(M_{Z}\right)$ [15]. Most of the $\chi^{2}$ contributions are from $R\left(\Gamma_{b \bar{b}} / \Gamma_{h a d}\right)$ and to a lesser degree from $R\left(\Gamma_{c \bar{c}} / \Gamma_{h a d}\right)$ and $A_{F B}^{0, l}$. Fig. 2 shows how $M_{W}$ changes with $m_{t}$ for fixed $m_{H}$ from the consistency of the full EWRC, on which the new world average $M_{W}$ and CDF $m_{t}$ along with the Best.fit solutions (depicted by $\diamond$ points) are also shown. The central values of the world average $M_{W}$ and CDF $m_{t}$ are consistent with a Higgs scalar mass about $1000 \mathrm{GeV}$, though $m_{H}=100 \mathrm{GeV}$ is within $1 \sigma$ because of large errors in the data. Clearly a better precision measurement of $M_{W}$ is desired to distinguish different $m_{H}$. For example, a change of $m_{H}$ by $200 \mathrm{GeV}$, i.e., from $400 \mathrm{GeV}$ to $200 \mathrm{GeV}$ at $m_{t}=174 \mathrm{GeV}$, results a change of $50 \mathrm{MeV}$ in $M_{W}$, i.e., from $80.30 \mathrm{GeV}$ to $80.35 \mathrm{GeV}$, as one can see from Fig. 2 and Fig. 3 . This in turn will require a precision of $11 \mathrm{GeV}$ or better in $m_{t}$ from the Best.fit curve in Fig. 1(a), which is consistent with the most statistical error improvement that may be achieved at the Fermilab Tevatron. Present precisions in the data entail a theoretical uncertainty of about $36 \mathrm{MeV}$ in $M_{W}$ which is about the overall error improvement expected at LEP-200.

We have examined the results of the minimal $\chi^{2}$-fits to the precision measurements of the Z-decay parameters at LEP with the aid of a modified ZFITTER program containing the full one-loop and dominant two-loop EWRC. While the result of QBA might appear to be in agreement with the 1993 data within $2 \sigma$ level of accuracy [21,30], the new world average value of $M_{W}$ and updated 1994 LEP data definitely disfavor the QBA and support for the evidence of the nonvanishing weak correction. Furthermore, the CDF $m_{t}$ is a solution of the minimal $\chi^{2}$-fits to the 1994 data with a Higgs scalar mass about $400 \mathrm{GeV}$. 
However this $m_{t}$ value can be shifted by as much as $8 \mathrm{GeV}$ due to the overall uncertainties in $\alpha_{s}\left(M_{Z}\right)$ and $\alpha\left(M_{Z}\right)$ for the moment and accordingly $m_{H}$ ranging $250-690 \mathrm{GeV}$. Further precision measurement of $M_{W}$ can provide a real test of the standard model as it will give a tight constraint for the needed amount of the EWRC and can provide a profound implication for the mass of t-quark and Higgs scalar. If $M_{W}$ is determined to within a 40 $\mathrm{MeV}$ uncertainty, $\Delta r$ within the context of the standard model will be tightly constrained to distinguish the radiative corrections and the $\chi^{2}$-fit to the Z-decay data with the 1994 accuracy can discriminate the mass range of the t-quark and Higgs scalar within $8 \mathrm{GeV}$ and $200 \mathrm{GeV}$ respectively, providing a crucial test for and even the need of new physics beyond the standard model. Finally if $M_{W}$ is determined to be larger than $80.31 \mathrm{GeV}$ with better than a $20 \mathrm{MeV}$ accuracy by the future precision measurements (perhaps reachable at LHC), this would be a definite sign for new physics beyond the standard model.

Note added: Most recent CDF measurement is $M_{W}=80.41(18) \mathrm{GeV}$ [31]. We note that the theoretical prediction of $M_{W}, 80.29(4)(2) \mathrm{GeV}$, is about $0.67 \sigma$ below the central value of the new experimental $M_{W}$.

\section{Acknowledgements}

One of us (KK) would like to thank the Center for Theoretical Physics, Seoul National University (CTPSNU), the Korea Advanced Institute of Science and Technology (KAIST) and LPTPE, Université P.\&M. Curie where parts of the work were done, for the kind hospitality during his sabbatical stay. Also the authors would like to thank Professors Hi-sung Song, Jae Kwan Kim, R. Vinh Mau and other colleagues at CTPSNU, KAIST, and LPTPE for the stimulating environment and supports and in particular Professor M. Lacombe for checking the numerical computations and to Professor J.E.Kim for his encouragements.

\section{References}

1. ALEPH Collab., D. Buskulic et al., CERN-PPE/93-40 (1993).

2. DELPHI Collab., D. Aarnio te al., Nucl. Phys. B367 (1911) 511.

3. L3 Collab., O. Adriani et al., CERN-PPE/93-31 (1993).

4. OPAL Collab., P. D. Action et al., CERN-PPE/93-03 (1993).

5. The LEP Collab., CERN-PPE/93-157 (1993). 
6. C. DeClercq; V. Innocente; and R. Tenechinl, in: Proc. XXVIIIth Rencontres de Moriond (Les Arcs, 1993).

7. L. Rolandi, in: Proc. XXVI ICHEP 1992, CERN-PPE/92 -175 (1992); M. P. Altarelli, talk given at Les Rencontres de Physique de la Vallee d'Aoste (La Thuil, 1993), LNF93/019(p); and J. Lefranceis, in: Proc. Int. EPS. Conf. H. E. Phys. (Marseille, July 1993).

8. F. Dydak, in: Proc. 25 Int. Conf. H. E. Phys., Eds. K. Phua, Y. Tamaguchi (World Scientific, Singapore, 1991), p3.

9. W. J. Marciano, Phys. Rev. D 20 (1979) 274; A. Sirlin, Phys. Rev. D22 (1980) 971; D29 (1984) 89; A. Sirlin and W. J. Marciano, Nucl. Phys. B189 (1981) 442; and A. Sirlin, NYU-TH-93/11/01. See also W. Hollik, in: Precision Tests of the Standard Model, ed. P. Langacker (World Scientific Pub., 1993); G. Altarelli, in: Proc. Int.EPS. Conf. H. E. Phys. (Marseille, July 1993); K. Hagiwara, S. Matsumoto, D. Haidt and C. S. Kim, KEK-TH-375, to be published in Z. Phys. C; and J. Erler and P. Langacker, UPR-0632T.

10. R. M. Keup, Fermilab-Conf-94/282-E; C. K. Jung, in: Proc. 27th ICHEP (Glasgow, July 1994).

11. P. Clarke; Y. K. Kim; B. Pictrzyk; P. Siegris; and M. Woods, in: Proc. 29th Rencontres de Moriond (Meribel,1994); R. Miquel, in : Proc. 22nd INS Symposium (Tokyo, March 1994); and D. Schaile, in: Proc. 27th ICHEP (Glasgow, 1994).

12. F. Abe et al., Phys. Rev. Lett. 73 (1994) 225; Phys. Rev. D50 (1994) 2966; S. Abachi et al, Phys. Rev. Lett. 72 (1994) 2138; FERMILAB-PUB-94/354-E.

13. F. Abe et al., FERMILAB-PUB-95/022-E; S. Abachi et al., FERMILAB- PUB95/028-E.

14. B. A. Kniehl, Int.J.Mod.Phys.A10 (1995) 443.

15. B. H. Smith and M. B. Voloshin, UMN-TH-1241/94; S. Franchiotti, B. Kniehl and A. Sirlin, Phys. Rev. D 48 (1993) 307; M. Shifman, TPI-MINN-94/42-T.

16. K. G. Chetyrkin, J. H. Kuhn, and A. Kwiatkowski, TTP94-32 and hep-ph/9503396.

17. Z. Hioki, Z. Phys. C49 (1991) 287; Phys. Rev. D45 (1992), 1814 ;Mod. Phys. Lett. A7 (1992) 1009; K. Kang, in: Proc. 14th Int. Workshop Weak Interactions and 
Neutrinos (Seoul, 1993), Brown-HET-931 (1993); and K. Kang and S. K. Kang, in:

Proc. Workshop on Quantum Infrared Physics (Paris, June 1994), Brown-HET-968 and SNUTP-94-97.

18. V. A. Novikov, L. B. Okun and M. I. Vysotsky, Mod. Phys. Lett. A8 (1993) 5929. See, however, CERN-TH-7214/94 for more recent analysis.

19. D. Bardin et al., CERN-TH-6443-92 (1992).

20. T. Takeuchi, A. K. Grant and M. P. Worah, FERMILAB-PUB-94/303-T.

21. K. Kang and S. K. Kang, in : Proc. Beyond the Standard Model IV (Granlibakken, Lake Tahoe, 1994), Brown HET-979 and SNUTP-94-128 (December, 1994).

22. F. Jegerlehner, PSI-PR-91-08 (April 1991).

23. Particle Data Group, Review of Particle Properties, Phys. Rev.D 45, No.11, Part II (1992).

24. CDF Collab., F. Abe et al., Phys. Rev. D 43 (1991) 2070.

25. W. Hollik (Ref. 7), p49.

26. K. Kang (Ref. 17); and Z. Hioki, Mod. Phys. Lett. A 10 (1995) 121.

27. T. Hebbeker, Aachen preprint PITHA 91-08 (1991); and S. G. Gorishny, A. L. Kataev and S. A. Larin, Phys. Lett. B 259 (1991) 144; and L. R. Surguladze and M. A. Samuel, Phys. Rev. Lett. 66 (1991) 560.

28. See, for example, W. Hollik (Ref. 9); CERN Yellow Book CERN 89-08, vol.1, p45 ; and K. Kang(Ref. 17).

29. M. Consoli and W. Hollik, in Z Physics at LEP 1, Vol. 1, eds. G. Altarelli et al., CERN 89-08 (1989).

30. See also K. Hagiwara, et.al. (Ref.9).

31. F. Abe et al., Phys. Rev. Lett. 75 (1995) 11. 


\section{Figure Captions}

Fig. 1(a) : The mass ranges of $m_{t}$ and $m_{H}$ from the minimal $\chi^{2}$-fit to the updated 1994 LEP data for $\alpha_{s}\left(M_{Z}\right)=0.123(6)$ and $\alpha^{-1}\left(M_{Z}\right)=128.87(12)$. The range of the Best.fit solutions (see the text) due to the error $\Delta \alpha_{s}= \pm 0.006$ is also indicated by the curves $\mathrm{A}$ and $\mathrm{B}$.

Fig. 1(b) : The mass ranges of $m_{t}$ and $m_{H}$ from the minimal $\chi^{2}$-fit to the updated $1994 \mathrm{LEP}$ data for $\alpha_{s}\left(M_{Z}\right)=0.123(6)$ and $\alpha^{-1}\left(M_{Z}\right)=128.87(12)$. The range of the Best.fit solutions due to the error $\Delta \alpha^{-1}\left(M_{Z}\right)= \pm 0.12$ is also indicated by the curves a and b.

Fig. $2: M_{W}$ versus $m_{t}$ for fixed values of $m_{H}$ from the full radiative correction in the standard model. The case of the minimal $\chi^{2}$-fit to the updated 1994 LEP data are indicated by $\diamond$.

Fig. 3 : $M_{W}$ versus $m_{H}$ for $m_{t}=174 \pm 16 \mathrm{GeV}$ : The middle region bounded by the solid lines represent the predicted theoretical value of $M_{W}$. Notice that as $m_{t}$ becomes heavier than $174 \mathrm{GeV}$ the lower bound of $m_{H}$ increases above $300 \mathrm{GeV}$ from the predicted $M_{W}$. 